Revue d'histoire de l'Amérique française

FB. REVUE D.HISTOIRE DE L'AMÉRIQUE FRANÇAISE

\title{
À la recherche de la nation : Maurice Séguin (1918-1984)
}

\section{Jean-Pierre Wallot}

Volume 38, numéro 4, printemps 1985

URI : https://id.erudit.org/iderudit/304308ar

DOI : https://doi.org/10.7202/304308ar

Aller au sommaire du numéro

Éditeur(s)

Institut d'histoire de l'Amérique française

ISSN

0035-2357 (imprimé)

1492-1383 (numérique)

Découvrir la revue

Citer cet article

Wallot, J.-P. (1985). À la recherche de la nation : Maurice Séguin (1918-1984).

Revue d'histoire de l'Amérique française, 38(4), 569-590.

https://doi.org/10.7202/304308ar d'utilisation que vous pouvez consulter en ligne.

https://apropos.erudit.org/fr/usagers/politique-dutilisation/ 


\title{
A LA RECHERCHE DE LA NATION: MAURICE SÉGUIN (1918-1984)
}

\author{
JEAN-PIERRE WALLOT \\ Département d' histoire \\ Université de Montréal
}

"La passion de l' histoire est celle d'une totalité, d'une globalité». (Denis Richet)

$\mathrm{Au}$ lieu de se complaire à triturer des faits d'importance faible ou moyenne, «l'historien de métier» devrait s'appliquer à «écrire l'histoire... d'une évolution politique, économique, culturelle ou sociale et d'en discuter, avec toute la liberté voulue, les composantes majeures». En effet, à quoi bon consacrer des années et des volumes méticuleux à débrouiller des faits de deuxième ou troisième ordre, si l'on élude «l'obligation scientifique d'étudier méthodiquement - sans se perdre dans les détails - en eux-mêmes et pour eux-mêmes, ces phénomènes de tout premier ordre, une fois connus les faits secondaires dont ils sont l'ultime expression»' . Ainsi pensait Maurice Séguin de l'historien et de sa discipline; ainsi pratiqua-t-il son métier exigeant jusqu'à sa mort, survenue le 28 août 1984. Bref, dès les années 1950, Séguin privilégiait «l'histoire forte» à la Lévis-Strauss, c'est-à-dire riche en explication et épurée des détails, par opposition à «l'histoire faible», c'est-à-dire descriptive et lourde de faits bruts ${ }^{2}$.

Voilà peut-être, rétrospectivement, l'apport essentiel de Maurice Séguin à l'historiographie canadienne et québécoise: la passion de la globalité, d'une prise en compte de l'évolution générale du Canada français, de ses origines à nos jours. Historien de synthèse, comme le qualifie si justement Jean Blain, il a fabriqué «de l'histoire vivante dont il peut sentir la résonnance et les répercussions dans la pensée et l'action d'un grand nombre de ses contemporains ${ }^{3}$. Jadis, Charles Péguy ne reprochait-il pas justement aux historiens leurs certitudes et leurs vérités apaisantes, leur histoire «usine frigorifique». Séguin ne s'est montré ni apaisant ni glacé ni antiquaire. Il jouissait de toute provocation intellectuelle et ses réparties cinglantes ont embroché plus d'un contradicteur imprudent.

Même s'il n'a pas occupé l'avant-scène des notables de l'histoire, Maurice Séguin a imprimé une direction nouvelle à l'historiographie

\footnotetext{
$1 \quad$ M. Séguin, La notion d'indépendance dans l'histoire du Canada/1965-1966 HC. 480 [Normes], polycopié, Université de Montréal, 1.

2 Voir G. Paquet et J.-P. Wallot, «Canada 1760-1850: anamorphoses et prospective», in R. Comeau, prés., Economie québécoise (Montréal, 1969), 300.

3 J. Blain, «Préface» à Maurice Séguin, La nation "canadienne» et l'agriculture (17601850) (Trois-Rivières, 1970), 21. A l'avenir, La nation.
} 
québécoise et canadienne. C'est lui qui a lancé et inspiré l'école historique dite de Montréal et le «néonationalisme». Son anonymat relatif ne l'a pas empêché d'influer considérablement sur la pensée nationale au Québec dans les années 1950-1980. C'est cette optique qui confère son sens au présent texte. Il ne s'agit ni d'un éloge de circonstance d'un mort célèbre ni d'une étude en profondeur d'une pensée touffue qui élude les simplifications. Tout au plus ai-je tenté un premier effort pour dégager les grandes arêtes de l'oeuvre de cet historien éminent, en évoquant d'abord l'homme, puis la genèse et le contenu de sa thèse de doctorat, qui rompait radicalement avec la pensée de son temps, et enfin ses «normes», sorte de distillation de sa pensée.

\section{I}

Maurice Séguin est né à Horse Creek, Saskatchewan, le 7 décembre 1918. Venu au Québec avec sa famille en 1921, il fréquente bientôt l'école Champlain et vit dans un quartier ouvrier (l'est de Montréal) où les commerces et les usines utilisent la langue anglaise avec leurs clients qui sont francophones à presque $100 \%{ }^{4}$. Son cours classique terminé au Collège Brébeuf, il obtient sa licence ès lettres classiques à la Faculté des lettres de l'Université de Montréal (1944), où il complète ensuite une thèse de doctorat en histoire en 1947. Puis, en 1948, suite à la démission de Lionel Groulx, Maurice Séguin devient professeur à l'Institut d'histoire de l'Université de Montréal et commence un décapage radical de la pensée nationaliste canadienne-française, d'abord auprès de ses collègues et de ses étudiants, puis, par irradiation progressive grâce à ses disciples, dans la société en général.

Pour le jeune homme que j'étais - c'était en 1954 -, Maurice Séguin apparaissait comme une «bête de scène». Excellent pédagogue, armé d'une force prodigieuse de rationalisation et de dialectique, tour à tour ironique, frondeur, irrespectueux des ancêtres et des autorités, provocateur auprès de son auditoire, il nous a cloués malgré nous, cohortes après cohortes, sur les bancs de l'incrédulité admiratrice. De sa voix assurée, il dévidait les arguments comme un feu roulant et la logique implacable dont il se bardait dans ses nombreuses batailles verbales, acculait les jeunes que nous étions sinon à la reddition mentale, du moins à une prise de conscience de notre infériorité relative: ignorance des faits (il semblait tous les connaître), incapacité d'articuler notre pensée à la même puissance.

Certes, les jeunes apprentis pouvaient toujours harceler le maître. Mais il ne désarmait pas, pendant des discussions aussi longues que fécondes, avec eux comme avec ses collègues Guy Frégault et Michel

\footnotetext{
$4 \quad$ M. Brunet, «Feu Maurice Séguin. Les étapes de l'historien et du maître à penser», [«Feu Maurice Séguin»], in Le Devoir, 8 septembre 1984, 11.
} 
Brunet, avec des professeurs d'autres facultés et d'autres courants de pensée (dont un groupe de juristes qui ont fait leur marque depuis) qui ont façonné, à leur heure, les cheminements diversifiés de la Révolution tranquille. Ces éclats d'esprit et ces joutes étincelantes ont ébloui plus d'une génération d'étudiants et, faut-il le dire, de collègues pourtant aguerris 5 .

Ce qui rebutait chez Séguin et suscitait parfois la révolte (toute intellectuelle) chez les étudiants, c'était sa froide assurance, son détachement presque clinique, lorsqu'il nous exposait le coeur de sa thèse: impossible assimilation, impossible indépendance, condamnation à la provincialisation, c'est-à-dire à la médiocrité perpétuelle. Mais sa disponibilité totale, son goût de la discussion, sa vitalité qui ne se démentira guère jusqu'aux graves maladies qui l'ont diminué physiquement à compter de la fin des années 1960, son respect et son affection mal dissimulée pour ses étudiants, lui gagnaient leur admiration et leur attachement. Peu enclin à la commission de conférences publiques et de publications aussi savantes qu'inutilement nombreuses, qui, à son avis, trahissaient souvent la pensée et semblaient toujours perfectibles, il concentrait toute son attention et son énergie à enrichir son enseignement, devenu, selon l'expression de Pierre Tousignant, «une épreuve initiatique» ${ }^{6}$.

A l'automne de 1961 et à l'hiver de 1962, j'ai partagé un bureau avec Maurice Séguin en attendant l'aménagement de l'Institut-Département en croissance dans de nouveaux locaux plus spacieux. Je préparais alors mes premiers cours et tentais de décanter les milliers de fiches que $\mathrm{j}$ 'avais rapportées des archives, afin d'en extraire une thèse de doctorat. A vrai dire, j'ai peu travaillé durant ces mois, malgré les heures insolites et trop longues de nos journées. En effet, le défilé d'étudiants et d'étudiantes ne cessait jamais: bien sûr, c'était Maurice Séguin qui les attirait, qui argumentait, expliquait, blaguait avec elles et eux. Il adorait les galéjades, et c'était peut-être dans ces moments que ce «grand timide» (l'expression est de Michel Brunet) se livrait un peu à son entourage à travers ce masque d'ironie et cette distance qu'il affectait. Il n'appela jamais son collègue Michel Brunet autrement que «Monsieur Brunet» ${ }^{7}$. J'ai été chanceux: avec le temps, ce fut «Wallot, vous...».

Le soir surtout, à la table de la cafétéria «Chez Valère» ou d'un très modeste restaurant des environs, ma femme et moi découvrions sa

\footnotetext{
5 M. Brunet a rappelé ces souvenirs dans «Feu Maurice Séguin» et dans «Guy Frégault: l'itinéraire d'un historien...», in P. Savard, prés., Guy Frégault (1918-1977) (Montréal, 1981), 32-39; J.-P. Wallot, «L'histoire, science de la vie», Présentation... à la Société royale du Canada (Ottawa, 1980), 35-39; idem, «L'histoire et la recherche du sens», Revue d' histoire de l'Amérique française, 37,4 (mars 1984): 534-535, 538.

6 P. Tousignant, «Maurice Séguin, maître à penser de l'école néonationaliste de Montréal [...]», Cahiers d'histoire, 5 (automne 1984): 19.

M. Brunet, «Feu Maurice Séguin», 14.
} 
culture très vaste, son goût pour certaines oeuvres littéraires, pour le cinéma, pour le théâtre. Ce furent des moments privilégiés, où j'appris beaucoup sur bien d'autres sujets que l'histoire et où Maurice Séguin se révéla l'homme de coeur et de sensibilité, presque chaleureux, qu'il était au fond de lui-même. Amitié que je pus ressentir plus tard, vingt ans après et malgré les dures condamnations qu'il m'avait adressées à l'occasion de mes «déviations» de commission et d'omission (adoption graduelle de perspectives différentes à compter du milieu des années 1960, intérêt pour des choses «secondaires» en histoire économique et sociale du Bas-Canada, telles la crise agricole, la monnaie, etc.; dispersion de mes énergies dans l'administration), lorsqu'il me pressa de revenir entièrement à l'histoire, peu de temps avant sa retraite.

On a beaucoup glosé sur l' «école de Montréal» et ses principaux protagonistes - «celui qui pense (Séguin), celui qui écrit (Frégault), celui qui crie (Brunet)» ${ }^{8}$. Séguin, soupçonne-t-on, aurait peu apprécié que ses collègues et amis répandent sa pensée sans lui en reconnaître publiquement la paternité pendant plusieurs années. En souffrit-il? Possiblement. Par contre, il considérait Frégault et Brunet comme des «étudiants» brouillons, «déviationnistes», trop pointus dans leurs interprétations de ses thèses fondamentales qui étaient très nuancées. Et puis, à l'instar de la majorité des historiens, ils lui semblaient trop publier, trop encombrer l'histoire d'oeuvres imparfaites, alors qu'il eût souhaité incarner l'essentiel de son apport en un livre modeste mais presque parfait. Par ailleurs, Michel Brunet a raconté comment Frégault refusait systématiquement de citer Séguin, de façon à l'aiguillonner à publier' .

Quoi qu'il en soit, l'esprit critique, voire féroce de Séguin, se tournait aussi contre lui-même avec la même rigueur implacable, de sorte qu'il n'était jamais satisfait d'un texte. Paradoxalement, les affrontements (verbaux) le stimulaient, de sorte qu'il ne redoutait pas la critique des autres. Travailleur infatigable, insomniaque d'habitude, il a amassé des masses de documents pour ses cours et préparé des éditions compliquées de textes pour ses étudiants, au grand dam des secrétaires ahuries. En réalité, il aurait voulu réussir une saisie totale et juste des principaux phénomènes de notre histoire avant de publier, d'où la minceur relative de son oeuvre écrite - et son projet de retraite: écrire enfin une synthèse séguiniste de «l'histoire des deux Canadas».

Autodidacte - il n'avait suivi qu'un cours d'histoire du Canada (celui de Groulx), mais avait beaucoup lu lorsqu'il opta pour un doctorat en cette matière -, il aiguisait la curiosité et l'esprit des étudiants, leur enseignait patiemment les vertus d'une vraie lecture et leur suggérait une multitude de sujets qu'il savait rendre plus passionnants les

$8 \quad$ Magazine MacLean, 6 (avril 1966).

9 M. Brunet, «Guy Frégault», 39. 
uns que les autres. Une fois la thèse choisie, il orientait le candidat vers quelques auteurs pertinents. Puis, il lui commandait de bien travailler «aux archives». Ses étudiants devaient alors se débrouiller comme luimême l'avait fait, sauf qu'il leur prodiguait encouragements et critiques, au moment de la prise des notes comme à celui de la rédaction des chapitres de thèse. Ainsi, comme tant d'autres étudiants avant et après moi, je n'aurais pu mener ma recherche de maîtrise à bien, n'eût été de l'aide concrète que me consentit Michel Brunet, après que le maître m'eût tracé le sujet de thèse et eût évoqué vaguement les «sources». Ce n'était pas de l'indifférence. Les détails, là aussi, le lassaient.

Maurice Séguin ne respectait que les esprits forts. Autant il contredisait, arguait, s'entêtait, autant il s'attendait naturellement à ce que ses étudiants agissent de même. Aussi était-il souvent éprouvé par les thèses de ses étudiants et disciples. On eût dit qu'il aurait préféré parfois ne disposer que des documents, tant l'analyse, dans la thèse, lui paraissait faible et inintéressante. Il respectait un point de vue autre que le sien, à condition qu'il fût articulé. Pierre Tousignant et moi-même avons goûté à ces séances de discipline, de dialectique, et y avons beaucoup appris.

Grâce à Jean Blain, à Pierre Tousignant et à Michel Brunet ${ }^{10}$, on connaît mieux aujourd'hui l'itinéraire intellectuel de Maurice Séguin. En 1944, il confie à Groulx, dont la forte personnalité l'a marqué, qu'il désire préparer une thèse de doctorat en histoire. Intéressé par le problème de l'infériorité économique des Canadiens français, insatisfait des réponses avancées par Étienne Parent, Errol Bouchette, Edouard Montpetit, Esdras Minville, voire Groulx lui-même, il s'inspire entre autres d'une conférence de Groulx prononcée en 1931 et intitulée «La déchéance incessante de notre classe moyenne», pour aborder le problème de l'agriculture et des Canadiens français dans le siècle suivant la Conquête. Il emprunte à Groulx les thèmes du «sol inaccessible» (rareté réelle ou spéculative) et du «sol improductif» (mauvaises techniques agricoles, épuisement des sols, crise agricole), notions qui ont conduit l'historien national à conclure que la crise agricole des années 1830 a entraîné la prolétarisation des campagnes, prélude à la prolétarisation urbaine au service de capitalistes étrangers.

Séguin développe alors des interprétations supplémentaires qui lui sont propres: entre autres, l'absence de débouchés stables, l'incertitude des marchés; la faible rémunération que peut apporter la terre à une paysannerie pauvre (il tente même de chiffrer ses revenus moyens).

$10 \quad$ Voir notes 3,5 et 6. 
Mais il comprend que le problème réside moins dans la crise agricole que dans le repliement agricole lui-même qui suit la Conquête, idée qu'il relie à une notion de colonisation intégrale empruntée à Esdras Minville $^{11}$ et à l'exemple des colonies américaines. Comme Séguin prend connaissance du texte de Minville en cours de rédaction de sa thèse, celle-ci se clôt, pour ainsi dire, par un rebondissement imprévu: les longs chapitres consacrés à l'agriculture canadienne et à la paysannerie, au marché, aux techniques agricoles, à l'inaccessibilité croissante des terres, débouchent sur une deuxième partie, beaucoup plus courte, qui soutient que le problème de l'agriculture s'avère secondaire par rapport à la question économique prise dans son ensemble.

A l'époque, la conclusion brise radicalement avec les interprétations traditionnelles: pas de salut de la nation par la Providence ou par la clairvoyance des élites laïques et religieuses; pas d'idéalisation du Régime français; pas d'apitoiement sur les destructions dues à la guerre; pas de triomphe ni d'égalité sous LaFontaine et la Confédération, etc. La Conquête n'apparaît plus comme une épreuve destinée à tremper et à épurer la nation canadienne-française; c'est un procès de déstructuration et de restructuration qui condamne le peuple canadien-français éventuellement minoritaire à l'infériorité socio-économique et politique, voire, à terme, à la provincialisation et à l'atrophie de sa culture.

La Conquête parque les Canadiens dans l'agriculture, écrit-il d'entrée de jeu. Auparavant, sous le Régime français, en période de colonisation intégrale, on constatait l'existence de l'esprit paysan, certes, mais aussi la présence, «chez beaucoup de Canadiens, [d']un esprit commercial et d'entreprise... Proportionnellement à leur nombre... les Canadiens comptèrent, par leurs explorateurs, leurs marchands, leurs coureurs de bois, parmi les grands hommes d'affaires de leur époque en Amérique» ${ }^{12}$. Or, après la Conquête, le repliement agricole constitue un «bien piètre moyen d'essor économique»: il ne peut que produire de petites épargnes dispersées, «juste de quoi faire vivre au jour le jour, dans un état voisin d'une honnête pauvreté un ensemble de petites fermes paysannes». En pratique,

...à cause du changement d'empire, les Canadiens étaient exclus du grand commerce. L'obligation de commercer avant tout à l'intérieur d'un monde étranger, la difficulté pour les Canadiens de

11 Minville s'était interrogé sur les raisons qui avaient amené les Canadiens français à une notion diminuée de la colonisation, soit de la colonisation de peuplement et de mise en valeur d'un territoire à la simple installation d'établissements agricoles sur des terres jusque-là inoccupées. La Conquête, explique-t-il alors, oblige les vaincus à se réfugier en eux-mêmes pour résister aux influences assimilatrices. «La population entière est donc refoulée vers la terre. Et la conquête du sol sera désormais et pour de nombreuses générations l'unique moyen de vie et d'expansion économiques. A partir de ce moment, coloniser, c'est essaimer d'une terre à l'autre... C'est sur cette notion diminuée - mais diminuée pour cause - de la colonisation que nous vivons encore.» (E. Minville, «La colonisation», L'agriculture (Montréal, 1943), 275ss).

12 M. Séguin, La nation, 53-54. 
nouer des relations avec les marchands inconnus de la nouvelle métropole ou des autres colonies, opposée à la grande facilité pour les Britanniques d'organiser entre eux les exportations et les importations, autant de causes qui se liguèrent d'abord pour annihiler le commerce extérieur que les Canadiens entretenaient, avant 1760 , et même pour les contraindre à délaisser une grande partie du commerce intérieur, principalement dans les branches étroitement liées au commerce extérieur... Des deux mentalités constatées chez les Canadiens avant 1760: l'esprit de commerce et d'entreprise pour une certaine classe et l'esprit paysan pour d'autres Canadiens, il ne subsistait plus que ce dernier. C'est alors que naquit la légende des Canadiens «paysans dans l'âme et terriens par vocation»! ${ }^{13}$

Les Canadiens deviennent des engagés au service de bourgeois étrangers qu'enrichit le commerce colonial. Désormais, à cause de la perte de leur métropole, ils ne pourront exploiter les ressources naturelles du pays à leur propre compte. Le succès individuel de quelques personnes ne peut masquer l'échec collectif. Les Canadiens survivront, en partie grâce à leur reflux sur la terre. Mais pour «une collectivité, il n'est pas donné de passer brusquement de la pauvreté paysanne à la grande industrie... [Il faut d'abord] passer par le commerce, afin d'accumuler les capitaux qui financeront ensuite l'industrie» 14 .

Séguin précise que les Canadiens ne pourront concurrencer les Britanniques dans l'érection des usines, la construction des chemins de fer, la création des banques, etc. Après la période commerciale, en effet, viendra, dans cette colonie «dite progressiste», l'explosion industrielle. Comme auparavant, aux Britanniques, «la direction et la propriété de la plupart des grandes entreprises, les Canadiens fourniront la main-d'oeuvre». C'est toujours la même infériorité: de commerciale, sous la première génération, elle devient financière, industrielle, commerciale, etc. Ce servage ne découle pas de mauvais traitements ou de l'imbécilité des vaincus, mais d'une «cause inévitable»: «l'occupation britannique, en elle-même, indépendamment des modalités de celleci». Pour éviter cette infériorité des Canadiens, il faudrait que

l'Occupant, pour ne pas leur nuire, se fasse violence afin de leur laisser une place proportionnée à leur importance numérique, qu'il dédaigne les bases stratégiques de Québec et de Montréal et n'exploite pas les ressources de la colonie, pourtant les plus accessibles à l'époque, mais réserve cette zone aux Canadiens pour le jour où ils pourront eux-mêmes la faire valoir. Un tel égard aurait équi-

13 Ibid, 245-246. «Pour Séguin, c'est le métier d'agriculteur lui-même qui dans le contexte géo-économique de la vallée du Saint-Laurent confine non à la «déchéance», mais à une médiocrité séculaire qui plonge ses racines au coeur même de la Nouvelle-France du XVIIe siècle et qui de toute façon, conquête ou pas, devait servir de bouillon propice à la prolétarisation urbaine.» (J. Blain, op. cit., 24).

14 M. Séguin, La nation, 247. 
valu, pratiquement, à rétrocéder aux Canadiens, non seulement leur territoire, mais aussi de vastes libertés politiques et davantage encore. C'eût été pour les Britanniques, ériger eux-mêmes une barrière étrangère à leur empire nord-américain, venant compliquer la colonisation du Haut-Canada et de l'intérieur. ${ }^{15}$

Les Britanniques, ajoute Séguin, ne peuvent exploiter leur conquête et soigner leurs propres intérêts sans fatalement bouleverser la vie économique des Canadiens et en faire des «serviteurs». Toutefois, le repli agricole amortit temporairement le coup. La masse ne le sentira que lorsqu'elle deviendra prolétaire, lors de l'industrialisation.

En plus du malaise agricole après 1820 , les Canadiens souffriront, ce qui est plus grave et existe depuis la Conquête, de «substitution parce que l'envahisseur les paralyse et accomplit, à leur place, le développement intégral du Québec « ${ }^{16}$. La mentalité paysanne et anticommerciale des Canadiens, devenue vraie jusqu'à un certain point, est due à la Conquête et aux Britanniques eux-mêmes: non à des actes de persécution, mais à leur existence comme colonisation intégrale au-dessus de celle, brisée, des Canadiens français. En parquant les Canadiens dans la seule agriculture, la Conquête affecte peu à peu leur pensée: «La Conquête leur a légué une conception diminuée de la vie économique.» ${ }^{17}$ D'où les illusions subséquentes des Canadiens: par exemple, la suppression des obstacles à la colonisation des terres comme remède à leur infériorité économique, etc. Mais même si les Canadiens avaient compris, soutient Séguin, rien n'aurait changé dans leur infériorité de fait $^{18}$. Par ailleurs, dans la lutte de races qui suit 1763, Londres doit choisir, ce qu'il fait en 1840. «Cependant, il ne faut pas oublier qu'une résistance paysanne même limitée, des circonstances heureuses (telle la Révolution américaine) et des erreurs de la part de l'occupant (le séparatisme de 1791 , le maintien du régime seigneurial, le fiasco de la colonisation agricole britannique, etc.), ont permis aux Canadiens de s'assurer le contrôle au moins démographique du pays de Québec dont pourtant ils n'habitaient pas, à la Conquête, la vingtième partie.» ${ }^{19}$

Ainsi se trouve campée, en quelques pages rapides, l'esquisse de ce qui deviendra peu à peu davantage normatif, concrétisé dans un cours de Normes, et de ce l'on pourrait considérer comme le point de départ de l'interprétation néonationaliste: la Conquête et ses consé-

15 Ibid, 250-251.

16 Ibid, 253-254. C'est nous qui soulignons.

7 Ibid, 256. Séguin trouve «des éléments fondamentaux (mêlés à des scories) dans ...Minville...: développement économique normal en Nouvelle-France déterminant une structuration sociale appropriée; démolition par la Conquête des structures économiques (réduites à l'agriculture) et sociales (réduites à la paysannerie et à l'élite cléricale) de la collectivité canadienne, accompagnée d'une dégénérescence des structures mentales qui amène à rechercher inconsciemment un idéal amoindri, frelaté». (J. Blain, op. cit., 27-28).

18 M. Séguin, La nation, 259-262.

19 Ibid, 263-264. 
quences structurelles, la subordination politique, économique et culturelle des Canadiens français au pays du Québec; la fin des virtualités collectives ${ }^{20}$ de la première colonisation qui, comme les colonies américaines, aurait pu évoluer normalement, dans un autre contexte, vers la constitution d'une nation française en Amérique du Nord; la provincialisation éventuelle, donc une survivance diminuée comme appendice dans un Canada progressiste et dirigé par la nation canadian ${ }^{21}$. A partir et au-delà du problème agricole des Canadiens français entre 1760 et 1850 , Séguin dégage «une interprétation globale, solidement articulée de l'histoire du Canada où l'on retrouve, liés les uns aux autres, les facteurs d'économie, de politique et de mentalité» ${ }^{22}$. La pensée trop tranchée de Frégault («il n'existe plus de Canada») ${ }^{23}$, qui pousse bien au-delà de la notion de médiocre survivance provinciale à perpétuité, et le braquage de l'éclairage sur la «déchéance de la bourgeoisie canadienne-française» par Michel Brunet ${ }^{24}$, combinés à l'effacement public de Séguin, même chez les historiens, ont fait dériver les débats sur des questions souvent secondaires par rapport à la vision globale de ce dernier.

La crise ne résidait pas uniquement à l'intérieur de l'agriculture. L'explication de l'exode rural, il faut la chercher bien plus loin que dans [une] «terre peu rémunératrice, inaccessible et improductive». Le grand drame de l'histoire économique des Canadiens n'est pas seulement d'avoir été bloqués dans leur expansion agricole ou d'avoir été un peuple de terriens à qui la terre a manqué... Les Canadiens formaient une nation pour laquelle le développement économique intégral était de règle comme pour toute autre nation. Ce qui leur a manqué, ce n'est pas seulement le sol arable, mais aussi les autres ressources de leur propre territoire avec la possibilité de les exploiter commercialement, l'accès aux capitaux assimilables et le pouvoir de s'industrialiser eux-mêmes, à leur propre compte. $^{25}$

En 1947, voire en 1950 ou 1960, on ne perçut pas les brisures profondes, dans la thèse de Maurice Séguin, avec l'historiographie

20 Jean Blain a déjà traité de la conception organiciste ou «organique» de la colonisation que l'on retrouve dans l'École de Montréal, notamment chez Frégault. Voir «Économie et société en Nouvelle-France: l'historiographie des années 1950-1960 - Guy Frégault et l'école de Montréal», Revue d' histoire de l'Amérique française, 28,2 (septembre 1974): 163-186.

21 En particulier, comme le note Blain, pour Séguin «c'est finalement au niveau des structures mentales que l'effet le plus pernicieux et aussi le plus durable de la Conquête se fait sentir». («Préface», 30).

22 Ibid, 31

23 G. Frégault, La société canadienne sous le Régime français (Ottawa, Société historique du Canada, 1954), 15.

${ }_{24} \mathrm{M}$. Brunet, La présence anglaise et les Canadiens. Études sur l' histoire et la pensée des deux Canadas (Montréal, Fides, 1954), 15-112.

${ }_{25}$ M. Séguin, La nation, 253-254. 
traditionnelle ${ }^{26}$. Même son apport important à l'histoire rurale fut ignoré par les historiens qui, pour la plupart, n'en prirent connaissance qu'à sa publication, en 1970. D'aucuns se sont contentés de se reférer au bref résumé que j'en avais publié dans la Revue d'histoire de l'Amérique française en $1966^{27}$, plutôt que de recourir à l'original. Finalement, la thèse parut en 1970, aux Editions du Boréal Express, sous le titre La nation "canadienne» et l'agriculture. Aujourd'hui, malgré les développements importants survenus en histoire agraire du Québec, on cite encore cet ouvrage rédigé il y a plus de $35 \mathrm{ans}^{28}$. C'est que la thèse comportait plusieurs notions et approches nouvelles: le marché, les revenus de la terre, la superficie fertile dans la zone seigneuriale, etc. Par contre, elle réitérait les thèses traditionnelles sur la mentalité des paysans canadiens, leurs méthodes de culture présumément mauvaises et routinières, bien qu'elle cherchait à relier cette vision au contexte socio-économique (absence de débouchés, coûts de la main-d'oeuvre, etc.)

\section{III}

L'essentiel, on l'a vu, n'était pas là. Les conclusions reposaient toutes sur une série de postulats tournant autour de la «normalité» d'une collectivité, idéalement incarnée, selon Séguin, dans l'État-nation, actuel ou virtuel. De 1947 à 1964-65, Maurice Séguin a élaboré une série de concepts pour comprendre la société en général et la nation en particulier. Il les qualifiait de «normes«. L'essentiel en était déjà explicité vers 1954-1957. Par la suite s'y ajoutèrent diverses nuances et précisions ainsi qu'une meilleure articulation des parties. Les Normes furent distribuées aux étudiants sous forme de polycopié vers $1965^{29}$. Déjà, en 1956, l'auteur en avait livré une brève esquisse à la Société historique du Canada ${ }^{30}$. Puis, il en avait tiré une interprétation rapide de l'évolution historique de l'idée d'indépendance sous forme de trois conférences diffusées sur les ondes de Radio-Canada, en mars et avril 1962.

\footnotetext{
26 M. Brunet, «Guy Frégault», 32-35 et Le Devoir, 8 septembre 1984; J. Blain, «Préface», 25 ss. Ce dernier souligne que ce qui a desservi l'enseignement de Séguin, ce furent moins les contradictions que l'ignorance de sa pensée chez les historiens et autres spécialistes: Séguin se situait dans une longue lignée d'historiens nationalistes dont le nationalisme déclamatoire avait lassé les esprits. En outre, Séguin ne diffusait pas sa pensée par l'écrit. De sorte qu'alors même qu'on l'incluait volontiers dans la lignée des répétiteurs, «c'est précisément l'historiographie nationaliste traditionnelle qui allait constituer pour le jeune chercheur, lui-même logicien du nationalisme, l'adversaire le plus coriace, c'est-à-dire celui qui vous encombre...», 31-32.

27 Voir Revue d' histoire de l'Amérique française, 20,3 (décembre 1966): 494ss.

28 V.g. voir R. M. McInnis, «A Reconsideration of the State of Agriculture in Lower Canada in the First Half of the Nineteenth Century», in D. H. Akenson, prés., Canadian Papers in Rural History (Gananoque, 1982), 3: 9-49; aussi J. McCallum, Unequal Beginnings: Agriculture and Economic Development in Quebec and Ontario until 1870 (Toronto, 1980), passim.

${ }_{29}$ C'est la version de 1965-1966 qui est utilisée ici sous le titre Normes (absent de la page couverture miméographiée).

${ }_{30}$ M. Séguin, «La notion d'indépendance dans l'histoire du Canada», résumé dans Société historique du Canada, Rapport de l'Assemblée annuelle, Ottawa, 1956, 83-85.
} 
Ces textes furent publiés d'abord dans la revue Laurentie ${ }^{31}$, avant de paraître, enrichis de notes, au Boréal Express en 1968 sous le titre de L'idée d'indépendance au Québec. Genèse et historique. Enfin, les notes de cours de Maurice Séguin ont inspiré plus d'une synthèse, dont celle de Denis Vaugeois ${ }^{32}$. L'auteur exposera sa propre synthèse dans une collection française qui n'a eu ici qu'une très faible diffusion ${ }^{33}$.

Le premier paragraphe de L'idée d'indépendance [...] synthétise les conclusions qui ressortent des 64 pages serrées des Normes, pages à simples interlignes et en style presque télégraphique.

L'annexion politique, dans une économie moderne et dynamique, entraîne inévitablement la subordination économique. L'infériorité politique et l'infériorité économique se conjuguent en s'aggravant. La culture elle-même, au sens le plus général du terme, intimement liée aux réalités politiques et économiques, est fortement perturbée au point qu'on ne peut même pas parler, pour le peuple minoritaire, de véritable autonomie culturelle. Pour cette école indépendantiste, l'indépendance politique complète est absolument nécessaire. Elle est à rechercher en elle-même comme un bien et elle est considérée comme un moyen irremplaçable pour assurer une maitrise suffisante de la vie économique et culturelle. ${ }^{34}$

On reconnaît, dans ce texte, l'interdépendance de toutes les facettes d'une société et le rôle primordial que joue, pour Séguin, l'instance politique dans la construction d'une colonie et son évolution vers la «maturité».

La thèse séguiniste, au coeur du néonationalisme, émerge, faut-il le préciser, en plein duplessisme. Qu'est-ce à dire? Au cours de ces années, le nationalisme traditionnel, encore dominant, persiste à célébrer le pacte égalitaire que serait la Confédération, la liaison languereligion-nationalité, le passé, le présent et l'avenir ruraliste, les lois, la langue et la religion comme constituant l'essence de la nation. Maurice Séguin participe pleinement au bouillonnement des idées qui, au coeur de transformations socio-économiques profondes, préparent la Révolution tranquille. Mais il ne s'attaque pas qu'au traditionalisme, mais à la notion prédominante et tronquée du national. Au cours de ces années 1945-1955/1960, les jeunes intellectuels, tributaires de différentes occupations et idéologies, sapent l'ordre établi, c'est-à-dire les valeurs agraires et étroitement religieuses, le nationalisme conservateur et défensif, l'immobilisme idéologique et social, la corruption et l'inaction du gouvernement provincial. Or, l'aspiration à la sécularisation et

\footnotetext{
31 Laurentie, 119 (juin 1962): 964-996.

32 D. Vaugeois, L'union des deux Canadas. Nouvelle Conquête? 1791-1840 (Trois-Rivières, 1962).

M. Séguin, «Le Québec» in Québec-Canada (Paris, 1973), coll. «L'humanité en marche», 22 (1973): 43-167.

34 M. Séguin, L'idée d'indépendance, 10.
} 
à la démocratisation de la société québécoise passe, pour la plupart, par le rejet du nationalisme, identifié au conservatisme et au duplessisme.

Séguin heurte ces conceptions de plein fouet en prônant la nécessité de la liberté collective tout autant que celle des libertés individuelles; en osant réfuter le mythe de l'égalité entre une majorité bien nantie et une minorité subordonnée au sein d'un même État fédéral et démocratique; en ridiculisant l'appel alors fréquent à l'héroïsme et au dépassement collectif permanent; en affirmant que la culture ne peut se dissocier de la vie globale d'une société, que la nation «normale» doit s'exprimer et se réaliser par un agir suffisamment autonome à tous les niveaux (politique, économique, social, culturel), que cette conduite par soi, en tout, dépasse de beaucoup en importance la manière de cet agir ou, en d'autres mots, que la langue et les coutumes qui distinguent comptent moins que ce qui pourrait rendre le Canada français semblable aux autres nations, à savoir une existence autonome, séparée. Or, autre point de friction, Séguin souligne simultanément la nécessité et l'impossibilité de l'indépendance - d'où l'épithète de «pessimiste» accolée à l'école néonationaliste par plusieurs spécialistes des sciences sociales. Dans ce cadre conceptuel, il reste aux Canadiens français du Québec (pas encore devenus des «Québécois») d'arracher la plus grande autonomie possible à l'intérieur de la Confédération comme remède partiel à une maladie incurable: la subordination, le remplacement, la diminution ${ }^{35}$.

Les Normes se présentent comme un ensemble de réflexions sur le phénomène national. Elles n'ont rien d'ethnocentrique, contrairement aux légendes qui circulent sur Séguin. Elles abordent plutôt de haut, sous forme de constats de sens commun et d'intuitions brillantes, la logique de la construction d'une nation, de toute nation, et des relations entre nations, en particulier dans des milieux composites où se côtoient une majorité et une minorité. Père du néonationalisme et sans doute de l'expression la plus achevée du nationalisme «intégral» canadien-français, Séguin expose systématiquement ses «normes», comme s'il élucidait les mystères d'un phénomène naturel lointain. Sa logique implacable érige un «modèle» explicatif qui veut rendre presque superflue une histoire détaillée des deux Canadas, tant il dessine clairement les lignes d'évolution.

L'introduction aux Normes insiste sur la nécessité de s'intéresser avant tout aux phénomènes primordiaux en histoire: en effet, cette approche constitue, «pour ceux qui ne sont pas des professionnels de l'histoire, la seule histoire importante et irremplaçable» (p. 1). Puis,

35 Voir J.-P. Wallot, «L'histoire et le néonationalisme des années 1947-1970», in G. Rocher et al., Continuité et rupture. Les sciences sociales au Québec (Montréal, 1984), 111-116; M. Brunet, «Mes années de formation, le révisionnisme de la décennie de 1950 et mes engagements», ibid, 45-50. 
elle passe en revue les interprétations «traditionnelles et majoritaires» (égalité politique dans un régime fédéral, inégalité corrigeable sur le plan économique, etc.) et les interprétations «traditionnelles... mais minoritaires» (possibilité d'obtenir l'indépendance politique, etc.) pour leur opposer une synthèse supprimant et jumelant des aspects des deux écoles. Cette synthèse nouvelle, nous avertit l'auteur, ne repose pas sur des documents nouveaux, mais sur une «confrontation plus rigoureuse des grands faits déjà connus de l'histoire des deux Canadas et... une application cohérente d'un système surveillé de normes concernant la façon de concevoir le déroulement de la vie politique, économique et culturelle d'une collectivité» (p. 3). Il n'y a là rien d'étonnant: tous les historiens choisissent les documents, les interprètent et reconstruisent le passé en fonction de normes inconscientes ou explicites, d'une vision du monde qui, étant inévitable, exige une explicitation. En ce sens, Séguin devance de quelques décennies ses collègues québécois et canadiens. L'introduction s'achève sur des considérations concernant l'objectivité, la sincérité et le respect de la vérité.

La première partie des Normes se déploie en plusieurs volets: vie et condition de vie (de l'homme et de la société en général), dynamique intégrale de la société (forces politiques, économiques, culturelles et leur interaction), sociologie du national (rapports entre les sociétés civiles), rapports entre le national et le social, rapports entre l'Église et l'État, formation des nations par la colonisation intégrale.

C'est dans le premier chapitre («vie et conditions de vie») que se trouvent définis plusieurs postulats ou concepts-clé qui sous-tendent toute l'interprétation séguiniste. Pour les individus comme pour les collectivités, vivre, c'est agir par soi, en collaboration avec les autres, mais dans l'autonomie. Une société est vivante dans la mesure où elle peut agir par soi comme collectivité.

L'AGIR (par soi) COLLECTIF est l'action concertée et organisée d'un certain nombre d'individus amenés à se grouper en société, à former équipe naturellement ou artificiellement, inconsciemment ou lucidement, volontairement ou involontairement à l'origine, spontanément ou par la force des choses, intégralement ou graduellement, et qui trouvent la liberté et les moyens d'exécuter, par une minorité ou par la majorité ou la totalité (de ces individus) dans leurs propres cadres, sous leur direction, grâce à leur initiative, les multiples activités qui constituent la fin de cette société. (p. 11)

En somme, affronter ses problèmes internes et externes, les résoudre soi-même, en tirer expérience, dynamisme, richesse d'être, liberté. Ce postulat est à la base de toute l'interprétation séguiniste et de ses Normes. Sans cette autonomie, c'est le remplacement par une autre collectivité dans son propre agir collectif, donc une «oppression essentielle«, second postulat qui découle du premier: l'agir par soi collectif permet l'enri- 
chissement, l'épanouissement de ses virtualités (intelligence, volonté, expérience, etc. $)^{36} \mathrm{La}$ «privation, le remplacement de l'agir (par soi) collectif équivalent à une oppression essentielle», distincte d'oppressions accidentelles (persécution, abandon, etc.)

Oppression essentielle: dès qu'une collectivité remplace, par son agir collectif, l'agir collectif d'une autre société, cette substitution ou ce remplacement (total ou partiel) est, ipso facto, diminution ou privation d'être, perte d'expérience, d'initiative et de possibilité d'accumuler des habitudes pour la collectivité remplacée. Cette substitution, en elle-même, indépendamment de ses modalités, constitue une oppression inévitable, nécessaire, essentielle, qui tient à la nature même de la vie, laquelle est agir (par soi). (p. 13)

Dans une société, chaque facteur, chaque aspect de la vie collective est essentiel à l'équilibre, à la santé de l'ensemble, et est donc irremplaçable. Ce troisième postulat de Séguin vise un certain nationalisme pour qui seule compte la culture, les affaires d'État et l'économie passant pour des champs d'action «neutres» susceptibles d'être partagés avec «l'autre» nation. Une sous-section traite ensuite de la dureté de la condition humaine (individuelle, collective); une autre, du «rendement limite» - «Il n'y a jamais de rendement collectif maximal... se méfier de la légende tenace de l'âge d'or...»-, de l'inégalité (individuelle, sociale, sociétale), de la prépondérance de la plus grande force. Sur ce dernier point, les constats de Séguin contredisent carrément l'idéalisme libéral alors en vogue dans Cité libre. «En histoire, on ne doit pas s'imaginer que l'inégalité et la lutte des individus, des classes et des nations (ainsi que la prépondérance de la force) auraient pu et pourraient être supprimées avec un peu d'intelligence, de bonne volonté... [Elles] sont l'essence même de la création, avant l'apparition de l'homme...» (p. 16). Une autre section récuse le déterminisme, mais circonscrit les limites de l'intervention de l'intelligence et de la volonté. Enfin, Séguin clôt le chapitre par un acte de foi en l'existence de «lois» régissant l'évolution des sociétés, d'une certaine permanence de la nature humaine.

Le chapitre II, l'un des plus intéressants et des plus modernes par son approche conceptuelle (voisine des schèmes marxiens), traite de la dynamique intégrale (interne) de la société. Il identifie sept facteurs (aspects démographique, géographique, économique, sociologique, politique, scientifique, artistique), qu'il ramène à quatre (le nombre, la richesse, l'organisation, la culture), puis à trois: l'économique, le poli-

36 Dans ses cours, Séguin utilisait l'exemple d'une équipe de hockey: ainsi, les Maples Leafs de Toronto jouant sur la glace et à la place des Canadiens de Montréal, au Forum. Les talents des joueurs de la seconde équipe, individuellement et collectivement, se trouveraient toujours là, toujours virtuels, mais ne pourraient jamais s'épanouir, même si les profits de la joute leur étaient versés. Parfois, Séguin utilisait aussi l'exemple du lion et du mouton dans un même pré, etc. 
tique, le culturel, les deux premiers constituant la «civilisation matérielle», le dernier, la «civilisation culturelle» (p. 18ss). De longs développements illustrent l'interaction entre ces différents facteurs dans toute société vivante et l'unité indispensable du processus vital de la société. Autre scandale à l'époque, il conclut que la vie, tout autant et même davantage que l'école, nourrit la culture et la dynamise. Certes, il faut éviter de surestimer ou de sous-estimer un des facteurs. Force exemples contemporains jaillissent sous sa plume: l'agriculturisme, la culture artistique, l'antiétatisme, le provincialisme «de ceux qui croient qu'une «nation» peut se contenter d'être province» (p. 24). Conclusion? «La société civile, une collectivité est un organisme «un» où l'on distingue des aspects intimement liés les uns aux autres... C'est un tout complexe, un réseau d'habitudes (expérience, initiative), de traditions, de capitaux, de techniques politiques, sociales, économiques et culturelles...» (p. 24).

Le chapitre III s'intitule «la sociologie du national». Il étudie les rapports entre les sociétés civiles ou les groupes ethniques. Séguin y définit la nation au sens général ${ }^{37}$, au sens étatique, au sens sociologique ou culturel, pour s'arrêter sur la nation au sens "INTÉGRAL». Celle-ci doit pouvoir posséder un agir (par soi) collectif dans tous les domaines, car il est «NÉCESSAIRE» dans tous ses aspects: il est un bien en soi tout comme l'oppression essentielle constitue un mal en soi (p. 26). D'où Séguin infère que «la MAITRISE de l'agir collectif l'emporte en valeur sur la MANIERE d'agir» (i.e. les lois, les moeurs, la langue, la religion, etc.) «Le plus IMPORTANT est ce qui est SEMBLABLE: pour toute nation, la maîtrise, l'agir (par soi) en pol., écon. et cult... Etre DISTINCT avant d'être DIFFERENT: il faut exister séparément d'abord (avant d'avoir une personnalité collective). Des nations distinctes ont la même langue ou les mêmes lois... La MANIERE d'être sort de l'EXISTENCE» (p. 27).

Après avoir défini différents types de nationalisme (politique, économique, culturel), Maurice Séguin en arrive à la définition suivante de ce phénomène «naturel», de cette tendance générale de toute société à tenter de maîtriser sa vie collective selon sa fin propre: «c'est - pour un groupe ethnique qui se reconnaît distinct - la recherche (s'il ne l'a pas), l'affirmation (s'il la possède), la défense (s'il la croit menacée), de la MAITRISE de sa vie politique, économique et culturelle.» (p. 27) Il s'agit donc d'un phénomène «constant» lié à la nature même de la vie organisée. «Tout nationalisme complet est séparatiste», mais non

\footnotetext{
37 «La NATION au sens le plus GÉNÉRAL: la nation ou nationalité est un groupe humain qui en est arrivé à se reconnaître DISTINCT pour de multiples raisons, très variables, pas toutes nécessaires à la fois, par exemple: une commune origine, selon la croyance acceptée et qui se perd dans le temps, une commune langue, des traditions communes ou une même histoire, l'occupation d'un même territoire, un impératif géographique, un accident historique, un partage dynastique ou un découpage impérialiste aux effets durables, etc...» (p. 25).
} 
isolationniste, puisque la coopération internationale s'effectue entre nations. Séguin ne peut s'empêcher, ici, de décocher quelques flèches aux historiens anglo-canadiens: «Il n'existe pas deux sortes de vrai nationalisme normal (maître chez soi), un $\langle$ broad $\rangle$ et un $\langle$ narrow nationalism> » (p. 27). Puis, l'auteur réfléchit sur les notions d'indépendance et de souveraineté, de nationalisme et de conservatisme, de patriotisme et de nationalisme.

Séguin distingue les accrochages entre nations ou les défaites superficielles, c'est-à-dire des défaites momentanées aux effets surmontables, de la «défaite fondamentale» (p. 30), quand «la nation vaincue ne peut plus, dans son ensemble ou dans sa majeure partie, retrouver la maîtrise de sa vie (pour toujours?)» (p. 30). Souvent, les nations existantes se sont façonnées dans le creuset de l'assimilation d'une multitude de collectivités qui auraient pu aspirer à leur indépendance et l'ont souvent recherchée. L'auteur étudie ensuite l'infériorité de voisinage, la subordination de voisinage («nation satellite»), la subordination sur place («nation annexée»). Cette dernière est «une communauté distincte (nation au sens sociologique) qui se sait distincte, qui a conscience de sa nationalité, mais qui est forcée «d'exister», de vivre tant bien que mal, comme minorité permanente, sous un système politique souverain dominé par un autre peuple majoritaire ou par un groupe d'autres peuples formant la majorité constante» (p. 32). Les types d'annexion peuvent aller de l'annexion politique à l'annexion économique ou aux deux.

Une longue section sur le fédéralisme analyse les diverses sortes de fédérations ou de confédérations. Puis, elle scrute le partage des compétences dans une union fédérale à deux. Dans l'optique indépendantiste de Séguin, le fédéralisme à deux, comme au Canada, entraîne une subordination constante de la minorité provincialisée: la majorité décide dans toutes les questions «nationales» et s'ingère constamment dans les affaires de la minorité provincialisée. «Il n'y a pas d'égalité possible entre la NATIONALITÉ MAJORITAIRE et la nationalité minoritaire dans TOUTE vraie UNION FÉDÉRALE: pas d'égalité de droits (de permissions), pas d'égalité de possibilités (de moyens).» (p. 35) C'est la majorité qui jouit de la souveraineté et qui agit par ellemême collectivement et majoritairement à tous les paliers: au gouvernement central et au plan régional. Pour la nation minoritaire, «il y a oppression essentielle appauvrissante (remplacement de l'agir collectif), directement (diminution de droits et de moyens, d'expérience, d'initiative, d'habitudes, etc.) et indirectement (à cause de l'interaction des facteurs, vie politique, économique et culturelle «provinciale»)» (p. 37).

Après diverses considérations, Séguin distingue les libertés individuelles de la liberté collective. Or, on ne peut démontrer scientifi- 
quement qu'il vaut mieux, pour les individus d'une collectivité donnée, que celle-ci soit indépendante ou annexée à une nation plus riche et plus évoluée. Et certes, «mieux vaut être bien annexé que mal annexé...» (p. 39). Mais être «annexé à une NATION INDÉPENDANTE, c'est quand même être annexé, être gouverné (partiellement ou totalement) par une autre nation, aussi longtemps que dure la conscience d'être une nation distincte. Sinon, indépendance et annexion seraient des synonymes» (p. 39). Enfin, l'annexion conduit à la provincialisation, à l'assimilation (bien plus l'agir par et dans l'autre que la perte de la langue, etc.), à moins que la collectivité ne se trouve «coincée», i.e. «ne pouvant accéder à l'indépendance, ne pouvant se fondre dans l'oubli de l'assimilation totale. Ces nationalités sont «condamnées» au supplice de la 'survivance'» (p. 40). Vivre comme un appendice ne peut engendrer une vie enrichissante. La nation minoritaire devient un milieu diminué, écartelé, appauvri, bien que des individus réussissent parfois à s'en sortir, généralement en s'assimilant à l'autre. Séguin traite ensuite de l'optique impérialiste, puis de l'optique fédéraliste, pour distinguer les diverses significations de l'assimilation et de l'annexion. Une dernière section se penche sur l'organisation internationale et ses implications pour les nations.

Le chapitre IV examine brièvement les relations entre le national et le social, deux aspects nécessaires de la vie d'une même communauté. Tout en reconnaissant l'existence de conflits sociaux et de conflits nationaux, Séguin rejette la prétention de tout réduire à des conflits sociaux et l'élimination des affrontements nationaux. «Une collectivité dominée par les chefs du prolétariat a autant besoin du national qu'une société capitaliste...» (p. 51) Le nationalisme ne peut être «la propriété de telle classe sociale» à travers le temps. Ce pan du schème séguiniste aurait eu avantage à s'enrichir d'apports extérieurs. C'est le moins développé.

Le chapitre $\mathrm{V}$ analyse les rapports entre le pouvoir civil et le pouvoir ecclésiastique, rapports qui ont marqué l'histoire du Québec au plus haut point, surtout aux $19 \mathrm{e}$ et $20 \mathrm{e}$ siècles: pouvoirs et prétentions de chacun des deux ordres, union ou séparation des deux puissances, etc.

Enfin, dans le chapitre VI, Séguin explicite les notions de colonisation d'exploitation et de colonisation de peuplement, les deux formes pouvant exister simultanément sur le même territoire. Puis, il aborde la seconde sous un triple aspect: 1) la cause ou la «nation-mère»; 2) l'association entre la cause et l'effet; 3) l'effet ou la «nation-fille». Dans la colonisation intégrale, «une nation, un tout organique intégral (gouvernement et peuple)... se projette à tous les paliers, dans tous ses rouages, pour former un autre tout organique intégral (gouvernement et peuple). Coloniser au sens normal du mot, c'est implanter, nourrir - protéger, 
défendre - développèr au moyen d'individus..., de cadres..., de richesses civilisatrices déjà accumulées dans la MÉTROPOLE (capitaux, techniques, outillages, savoir-faire administratif, militaire, culture intellectuelle, etc...)» (p. 55). Dans la colonie comme dans la métropole s'impose la même exigence: nécessité du nombre, de la richesse, de l'organisation politique, de la culture, bref d'un développement équilibré. D'une phase où la métropole est l'unique agent de colonisation, on passe à une phase où la colonisation est devenue oeuvre conjointe et enfin, à la phase où la colonie peut s'affirmer comme seul agent émancipé et autonome, comme nation indépendante (p. 57).

La seconde partie des Normes, beaucoup plus brève (p. 59-64), ne fait qu'appliquer à l'évolution historique canadienne les postulats et concepts énoncés dans les chapitres antérieurs. Ainsi, dans «l'optique nationaliste intégrale», la situation du Canada sous le Régime français n'est «pas idéalisée - mais revalorisée»:

C'est la seule époque de son histoire où le nationalisme (séparatiste) des Canadiens (français) s'enracine pleinement dans la réalité. Pendant plus de cent ans, les Canadiens (d'origine française) vivent seuls dans un État séparé... Aussi longtemps que ce Canada demeure «seul», aussi longtemps que la cause de sa naissance comme colonie et le garant de son épanouissement comme nation, la MÉTROPOLE FRANÇAISE, se tient derrière lui pour le protéger militairement, pour le coloniser avec ses hommes, ses capitaux, ses techniques, ses institutions, etc., ce CANADA est apte à devenir une NATION normale. (p. 59)

Certes, le Canada de 1700 ou même de 1750 ne constitue pas une nation moderne, complètement équipée. Il est une "colonie», sous la juridiction d'une «métropole NATURELLE et à distance. Ce pouvoir extérieur cesserait un jour nécessairement si la France réussissait sa colonisation» (p. 60). Le Canada a aussi besoin des capitaux, des colons, des techniques en provenance de la France ou d'ailleurs. La virtualité de l'obtention du statut de nation subsiste toujours. Mais cette colonisation doit résister aux pressions de la colonisation britannique, 20 fois plus peuplée, plus riche, plus puissante.

La Conquête, puis la Cession, «c'est la fin de la projection française: il n'y aura pratiquement plus de colons, de capitaux, etc. pour soutenir, alimenter directement cette jeune nation d'Amérique. Le Canada (français) subit une «désorganisation» politique et économique. Il n'y a plus d'État colonial franco-canadien. Il n'y a plus d'Économie coloniale franco-canadienne. Des Canadiens (fr.) continuent à exister sous une autorité politique anglaise. Des agriculteurs et des marchands canadiens (-fr.) survivent dans un système économique anglais» ( $p$. 62). Bien plus, le Canada est soumis à une «AUTRE colonisation» (p. 63). Maintenant, c'est la projection et la protection par la Grande-Bretagne qui enfante, nourrit et défend un deuxième Canada, anglais celui- 
$\mathrm{là}^{38}$. Suffisamment enracinés, les Canadiens lutteront contre cette nouvelle colonisation et devront éventuellement, comme minorité annexée, apprendre à coexister avec et sous elle.

Au cours de ses 35 années d'enseignement, Maurice Séguin a non seulement enseigné ses Normes et brossé des tableaux synthétiques de l'histoire du Canada qui s'en inspiraient. Il a aussi accumulé une masse monumentale de documents divers, dirigé plusieurs dizaines de mémoires et de thèses, donné des cours et des séminaires parfois très spécialisés, où l'étude de la presse anglophone, du Rapport Durham ou de la correspondance des gouverneurs (par exemple, nous devions étudier dans la série des Documents constitutionnels publiés par les Archives publiques du Canada), permettait à cet historien un va-et-vient essentiel entre la problématique et les sources.

On peut regretter que Maurice Séguin ne se soit pas inséré dans le réseau des sciences sociales, n'ait pas confronté ses Normes à des schèmes conceptuels comparables ou plus généraux qui auraient pu enrichir le sien, le faire évoluer, le faire connaître. D'aucuns ont cru que son trop grand respect pour Groulx, à qui il devait son entrée à l'Université de Montréal, l'a empêché de contredire ouvertement son maître sur les plans conceptuel et historique. Groulx est mort en 1967. Mais Séguin souffrait déjà d'insomnie chronique et d'ulcères. Est-ce la raison de cet étrange renfermement scientifique qui contrastait tant avec son prosélytisme aguerri auprès de ses collègues immédiats et de ses étudiants? Était-ce sa timidité, sa répugnance à commettre des écrits inachevés et imparfaits? Chose certaine, Séguin n'a jamais redouté la contradiction: pendant des décennies, il a multiplié les affrontements intellectuels. En tout état de cause, ce furent d'anciens disciples, tels Gilles Bourque, Pierre Tousignant et d'autres ${ }^{39}$, qui fécondèrent le modèle original par des apports marxistes ou l'histoire comparée. D'autres ont retenu des dimensions majeures de son échafaudage conceptuel (la colonisation intégrale, les liens impériaux, l'infériorité structurelle), tout en se délestant de pans non moins importants de sa pensée et surtout de cette logique radicale, fermée, qui en venait presque à nier l'histoire en la faisant tenir toute entière dans quelques postulats généraux.

\footnotetext{
38 «C'est une catastrophe qui arrache cette jeune colonie à son milieu protecteur et nourricier et l'atteint dans son organisation comme peuple ou comme nation en formation. Le Canada français ne sera plus seul. Sur le même territoire, dans ce Québec même, naît une autre colonisation, anglaise cette fois, colonisation qui s'imposera dès l'origine par sa suprématie politique et économique et qui finalement consolidera ces suprématies par le nombre en devenant la majorité (vers 1840).» (p. 64)

39 G. Bourque, Classes sociales et question nationale au Québec, 1760-1840 (Montréal, 1970); P. Tousignant, La genèse et l'avènement de la Constitution de 1791, thèse de Ph.D., manuscrite, Université de Montréal, 1971; idem, «Problématique pour une nouvelle approche de la Constitution de 1791», Revue d' histoire de l'Amérique française, 27,2 (septembre 1973): 181234.
} 
Après ce bref tour d'horizon qui ne rend pas justice à la richesse et à la solidité du paysage intellectuel construit patiemment par Maurice Séguin durant 35 ans, on peut s'interroger sur la modernité de son oeuvre, sur sa pertinence aujourd'hui. Il est probable que l'orientation idéologique et politique actuelle, au Québec, repoussera dans l'ombre, du moins pour un temps, les notions fondamentales que Séguin a élaborées sur le nationalisme intégral: l'agir (par soi) collectif, l'annexion, l'oppression essentielle, etc. Curieusement, ces concepts, répandus dans les années 1960 par des enseignants et des penseurs politiques, n'ont guère servi lors du référendum de 1980: les débats, alors, ont effleuré les questions essentielles, s'accrochant à ce que Séguin aurait appelé les «accidents» (le bon ou le mauvais gouvernement, à Québec et à Ottawa, la tendance centralisatrice du gouvernement fédéral d'alors, la nécessité d'une seule politique sociale, etc.) Il est permis de penser qu'à long terme, l'approche conceptuelle, à la Séguin, rajeunie et renouvelée, aura un impact plus durable que les arguments politiques et partisans.

Fait plus important pour les historiens, Maurice Séguin a exposé des générations d'étudiants, pour la première fois, à un «modèle» historique, à un schéma conceptuel articulé et explicatif de l'histoire du Canada. L'explicitation de ses postulats, leur systématisation, voilà aussi une approche novatrice en son temps et encore aujourd'hui. A vrai dire, trop d'historiens refusent encore d'amorcer semblable démarche et de faire accéder leurs préjugés ou leurs préconceptions au stade de l'explicitation indispensable à toute démarche scientifique.

Dans son schéma, Séguin découpe la réalité sociale dans les trois grandes dimensions (l'économique, le politique, le culturel) qui correspondent, grosso modo, aux découpages utilisés par diverses écoles de pensée contemporaines. Toutefois, contrairement au marxisme, par exemple, il situe l'instance première au niveau politique, du moins dans la perspective d'une nation coloniale en construction. En ce sens, sa pensée ne pouvait que trouver des échos, des correspondances dans les projets des bâtisseurs de la Révolution tranquille et des concepteurs de l'État québécois contemporain.

Enfin, pour Séguin, l'histoire n'achevait jamais de s'étayer, selon la belle expression de Jean Blain ${ }^{40}$, de se raffiner, de se modifier. Il considérait que l'historien contemporain devrait «constamment faire appel, pour choisir, juger, retenir, coordonner et hiérarchiser les multiples faits, à des conceptions que lui fourniront les sciences politiques,

40 J. Blain, «Préface», 17. 
économiques, sociales, la géographie, à tout un ensemble de normes très vastes et jamais totalement maîtrisé ${ }^{41}$.

Maurice Séguin a été un véritable maître. Non au sens d'un dominateur qui se serait fait entourer de disciples complaisants et sidérés: il aimait trop la vie et la contradiction. Mais au sens où il a formé des centaines, voire des milliers d'étudiants qui ont distillé de sa pensée des éléments de compréhension du passé et du présent, une façon de considérer de manière critique leurs propres conceptions et celles des autres, au sens également d'un certain détachement face aux oppositions, d'une lucidité sans cesse en éveil et finalement, malgré les bourrasques occasionnelles, du respect et de l'admiration contenue face au travail sérieux de ses collègues, même ceux qui «déviaient» ou pensaient différemment de lui. Il est malheureux qu'il soit disparu avant d'avoir donné le fruit enfin mûr de ses longs travaux.

\section{NOTICE BIBLIOGRAPHIQUE SOMMAIRE DE MAURICE SÉGUIN}

«La Conquête et la vie économique des Canadiens», L'Action nationale, 28 (décembre 1946): 308-326. Ce texte a été reproduit dans R. Comeau, prés., Économie québécoise, 345-361 et dans R. Durocher et P.-A. Linteau, Le «retard» du Québec et l'infériorité économique des Canadiens français (s.1., Boréal Express, 1971), coll. «Études d'histoire du Québec, no 1, 93-111.

La nation "canadienne» et l'agriculture (1760-1850), thèse de doctorat présentée à la Faculté des Lettres de l'Université de Montréal, 1947, 274 pages, publiée sous le titre La nation «canadienne» et l'agriculture (1760-1850). Essai d'histoire économique (Trois-Rivières, Boréal Express, 1970), $284 \mathrm{p}$.

«Le régime seigneurial au pays du Québec, 1760-1854», Revue d'histoire de l'Amérique française, 1,3 (décembre 1947): 382-402; 1,4 (mars 1948): 519-532.

«La notion d'indépendance dans l'histoire du Canada», résumé de communication in La Société historique du Canada, Rapport de l'Assemblée annuelle (Ottawa, 1956), 83-85.

«Genèse et historique de l'idée séparatiste au Canada français», Laurentie, 119 (juin 1962): 964-996.

L'idée d'indépendance au Québec. Genèse et historique (Trois-Rivières, Boréal Express, 1967), 66 p. Une partie en a été reproduite dans J.-P. Bernard, Les rébellions de 1837-1838 (Montréal, Boréal Express, 1983), 173-189.

$41 \quad$ M. Séguin, Normes, 4. 
«Le Québec», dans Québec-Canada (Paris, Éditions du Burin), coll. «L'Humanité en marche», 22 (1973): 43-167.

\section{DOCUMENTS ET NOTES POLYCOPIÉS}

La notion d' indépendance dans l' histoire du Canada [ou Normes], notes polycopiées pour le cours Histoire du Canada 480, 1965-1966, 64 p.

Synthèse de l'évolution politique et économique des deux Canadas, notes polycopiées pour le cours Histoire du Canada 480, 1965-196?, $287 \mathrm{p}$.

Le Rapport Durham (traduction des principaux passages du rapport, avec le texte original en regard, document polycopié, de 1967 à 1970).

Documents sur le British North America, 1846-1848 (Montréal, Presses de l'Université de Montréal, 1973), 142 p. Première édition, 1962.

Extraits du journal Le Canadien, 1831-1842 (documents polycopiés qui se sont accrus avec les années).

Extraits de la Correspondance de Durham (documents polycopiés qui se sont accrus avec les années). 